\author{
Davide Chiumello \\ Antonella Marino \\ Matteo Brioni \\ Federica Menga \\ Irene Cigada \\ Marco Lazzerini \\ Maria C. Andrisani \\ Pietro Biondetti \\ Bruno Cesana \\ Luciano Gattinoni
}

\title{
Visual anatomical lung CT scan assessment of lung recruitability
}

B. Cesana

Sezione di Statistica Medica e Biomedica, Università di Brescia, Brescia, Italy

Published online: 19 September 2012

(C) Copyright jointly held by Springer and ESICM 2012

Electronic supplementary material

The online version of this article (doi:10.1007/s00134-012-2707-9) contains supplementary material, which is available to authorized users.

D. Chiumello $(\bowtie) \cdot$ L. Gattinoni Dipartimento di Anestesia, Rianimazione (Intensiva e Subintensiva) e Terapia del Dolore, Fondazione IRCCS Ca' GrandaOspedale Maggiore Policlinico, Via Francesco Sforza 35, 20122 Milan, Italy e-mail: chiumello@libero.it

Tel.: +39-2-55033237

Fax: +39-2-55033230

A. Marino - M. Brioni - F. Menga .

I. Cigada $\cdot$ L. Gattinoni

Dipartimento di Anestesiologia, Terapia

Intensiva e Scienze Dermatologiche,

Università degli Studi di Milano,

Milan, Italy

M. Lazzerini · M. C. Andrisani ·

P. Biondetti

Dipartimento di Radiologia, Fondazione IRCCS Ca' Granda-Ospedale Maggiore Policlinico, Milan, Italy
Abstract Purpose: The computation of lung recruitability in acute respiratory distress syndrome (ARDS) is advocated to set positive end-expiratory pressure (PEEP) for preventing lung collapse. The quantitative lung CT scan, obtained by manual image processing, is the reference method but it is time consuming. The aim of this study was to evaluate the accuracy of a visual anatomical analysis compared with a quantitative lung CT scan analysis in assessing lung recruitability. Meth-

ods: Fifty sets of two complete lung CT scans of ALI/ARDS patients computing lung recruitment were analyzed. Lung recruitability computed at an airway pressure of 5 and $45 \mathrm{cmH}_{2} \mathrm{O}$ was defined as the percentage decrease in the collapsed/ consolidated lung parenchyma assessed by two expert radiologists using a visual anatomical analysis and as the decrease in not aerated lung regions using a quantitative analysis computed by dedicated software.
Results: Lung recruitability was $11.3 \%$ (interquartile range 7.39-16.41) and $15.5 \%$ (interquartile range 8.18-21.43) with the visual anatomical and quantitative analysis, respectively. In the Bland-Altman analysis, the bias and agreement bands between the visual anatomical and quantitative analysis were $-2.9 \%(-11.8$ to $+5.9 \%)$. The ROC curve showed that the optimal cutoff values for the visual anatomical analysis in predicting high versus low lung recruitability was $8.9 \%$ (area under the ROC curve 0.9248, $95 \%$ CI 0.8550-0.9946). Considering this cutoff, the sensitivity, specificity, and diagnostic accuracy were $0.96,0.76$, and 0.86 , respectively. Conclusions: Visual anatomical analysis can classify patients into those with high and low lung recruitability allowing more intensivists to get access to lung recruitability assessment.

Keywords Lung CT scan .

ARDS - Mechanical ventilation .

Lung recruitability · PEEP .

Lung overdistension

\section{Introduction}

The recommended ventilator management in acute respiratory distress syndrome (ARDS) combines the use of a low tidal volume ventilation to avoid overstress- overdistension [1, 2], with an adequate level of positive end-expiratory pressure (PEEP) to avoid cycling opening/ closing and collapse of alveolar units [3].

Several methods have been suggested to evaluate the PEEP-induced lung recruitment [4]. Owing to its 
simplicity the multiple pressure-volume curve has been proposed as an adequate surrogate for the estimation of lung recruitment $[5,6]$. However, it may underestimate lung recruitment in patients with focal aeration loss [7]. Electrical impedance tomography provides noninvasive real-time imaging of pulmonary inflation which could predict the lung recruitability [8], but it computes only a small fraction of the lung and is affected by the movements of intrathoracic blood due to the application of PEEP. Recently, Bouhemad et al. [9] showed that PEEPinduced lung recruitment estimated by transthoracic lung ultrasound was related to the one computed by the pressure-volume curves.

Thus at the present time, quantitative lung CT scan analysis remains the reference method for computing PEEP-induced lung recruitment $[10,11]$. However, this analysis requires dedicated software and a manual delineation of the perimeter of the lungs in each CT image [10]. Thus, up to $6 \mathrm{~h}$ is often required to complete lung CT scan computation [12]. Consequently, because of the time required, quantitative lung $\mathrm{CT}$ scan analysis remains only a research tool. To increase the feasibility of using the lung CT scan as a clinical tool to estimate the PEEP-induced lung recruitment, in this study we evaluated the accuracy of a visual anatomical lung CT scan analysis compared to a quantitative lung CT scan analysis. This new analysis, which is based on the visual assessment of consolidated/ collapsed regions on CT lung images, does not require any dedicated software or a manual delineation of the lung. Thus, this simple bedside analysis could favor the increase of the use of lung CT scan as a reliable method to estimate the lung recruitability and to set PEEP for preventing the lung collapse.

\section{Patients and methods}

Fifty sets of two complete lung CT scans of ALI/ARDS patients were analyzed, randomly taken from a database of a multicenter study investigating lung recruitment [11] and from a database of unpublished data investigating the effect of PEEP (www.clinicaltrial.gov number INTC0068 2942). All patients met the standard criteria for ALI/ ARDS [13]. The exclusion criteria were an age of less than 16 years, pregnancy, and chronic obstructive pulmonary disease. The patients were studied at two university hospitals (Italy and Germany) after approval by the local institutional review boards according to the national regulations of each institution had been given.

\section{CT scan}

Sedated and paralyzed patients underwent two whole lung CT scans. The CT scanner was set with the following parameters: collimation $5 \mathrm{~mm}$, interval $5 \mathrm{~mm}$, bed speed $15 \mathrm{~mm} / \mathrm{s}$, voltage $140 \mathrm{kV}$, and current $240 \mathrm{~mA}$. The first lung CT scan was performed at an inspiratory plateau pressure of $45 \mathrm{cmH}_{2} \mathrm{O}$ during an end-inspiratory pause and the second one at a PEEP value of $5 \mathrm{cmH}_{2} \mathrm{O}$ during an end-expiratory pause. Baseline ventilation was set according to the clinical treatment. Ventilator settings were kept unchanged throughout the study.

In addition each patient was classified by the two radiologists as presenting a lobar or patchy distribution of consolidation/collapse in the lung [14].

\section{Visual anatomical lung CT scan analysis}

The consolidated/collapsed lung region appears, on lung CT images, as a zone of increased pulmonary attenuation in which both the margins of vessels and the airway walls are not visible [15]. The lung recruitability was computed as the difference in the percentage of consolidated/collapsed tissue passing from an airway pressure of 5 to $45 \mathrm{cmH}_{2} \mathrm{O}$, obtained as the mean of values assigned by two expert radiologists [11]. The lung CT images were visualized by using a freely available piece of software (Efilm workstation, Canada).

The two radiologists visually assessed the percentage of consolidated/collapsed region in each bronchopulmonary segment unaware of the quantitative lung CT scan analysis evaluation. A visual four-step scale (0-25, $25-50,50-75$, and $75-100 \%$ ) was used to quantify the percentage of the collapsed/consolidated tissue in each segment of the lung. The percentage of consolidated/ collapsed tissue of each bronchopulmonary segment was multiplied to the estimated volume percentage of each pulmonary segment with respect to the total lung volume [16]. The total consolidated/collapsed lung percentage was then obtained as the sum of the segment percentages.

Briefly, in the lung there are 18 bronchopulmonary segments which constituted the six lung lobes (right upper, medial, lower lobes, and left upper, lower, and lingula lobes) (see Electronic supplementary material for a more detailed description).

\section{Visual identification of bronchopulmonary segments}

On lung CT images, the bronchopulmonary segments are easily centrally identified by the distribution of their segmental bronchi, which arise from the lobar bronchus of the tracheobronchial tree [17]. The bronchopulmonary segments which constitute a single lobe were arbitrarily considered to have the same volume. Only axial scans were used for visual analysis.

In the right lung, in axial scan craniocaudally, the apical segment of the upper lobe was arbitrarily identified as the lung parenchyma between the lung apex and the 
visualization of the anterior and posterior segmental bronchi (Fig. 1a). The anterior (ventral half) and posterior (dorsal half) segments of the upper lobe were identified with their bronchus which course horizontally in the transaxial plane (Fig. 1b).

Caudally to the upper lobe, the medial (more ventral) and lateral segments (more dorsal) of the middle lobe are identified by the presence of their bronchi in the same transaxial plane (Fig. 1c). The superior segment of the inferior lobe is localized dorsally to the major right fissure and it extends inferiorly to the branching of the inferior lobe bronchus (Fig. 1c). Caudally to this point, the basal segments of the lower lobe extend to the diaphragm (Fig. 1d). The relative position of these segments is identified by the position of their segmentary bronchi which is quite stable. Reading anticlockwise they are the anterior, lateral, posterior, and medial segments (Fig. 1d).
In the left lung the apical-posterior segment was identified between the lung apex and the visualization of the apical and posterior bronchi (Fig. 1a). The anterior segment of the upper lobe is recognized in the ventral half of the axial scan in which both the apical and anterior segmental bronchi are visible (Fig. 1b). In the same CT scan the dorsal half represents the continuation of the apical-posterior segment (Fig. 1b).

The superior segment of the inferior left lobe is identified dorsally to the major left fissure, to the branching of the lower lobar bronchus (Fig. 1b, c). Craniocaudally the basal segments of left inferior lobe extend from the branching of the inferior bronchus to the diaphragm. Reading clockwise they are anterior medial, lateral, and posterior segments (Fig. 1d).

The lung parenchyma localized at the left of the heart chambers and anteriorly to the fissure is the lingula. Its

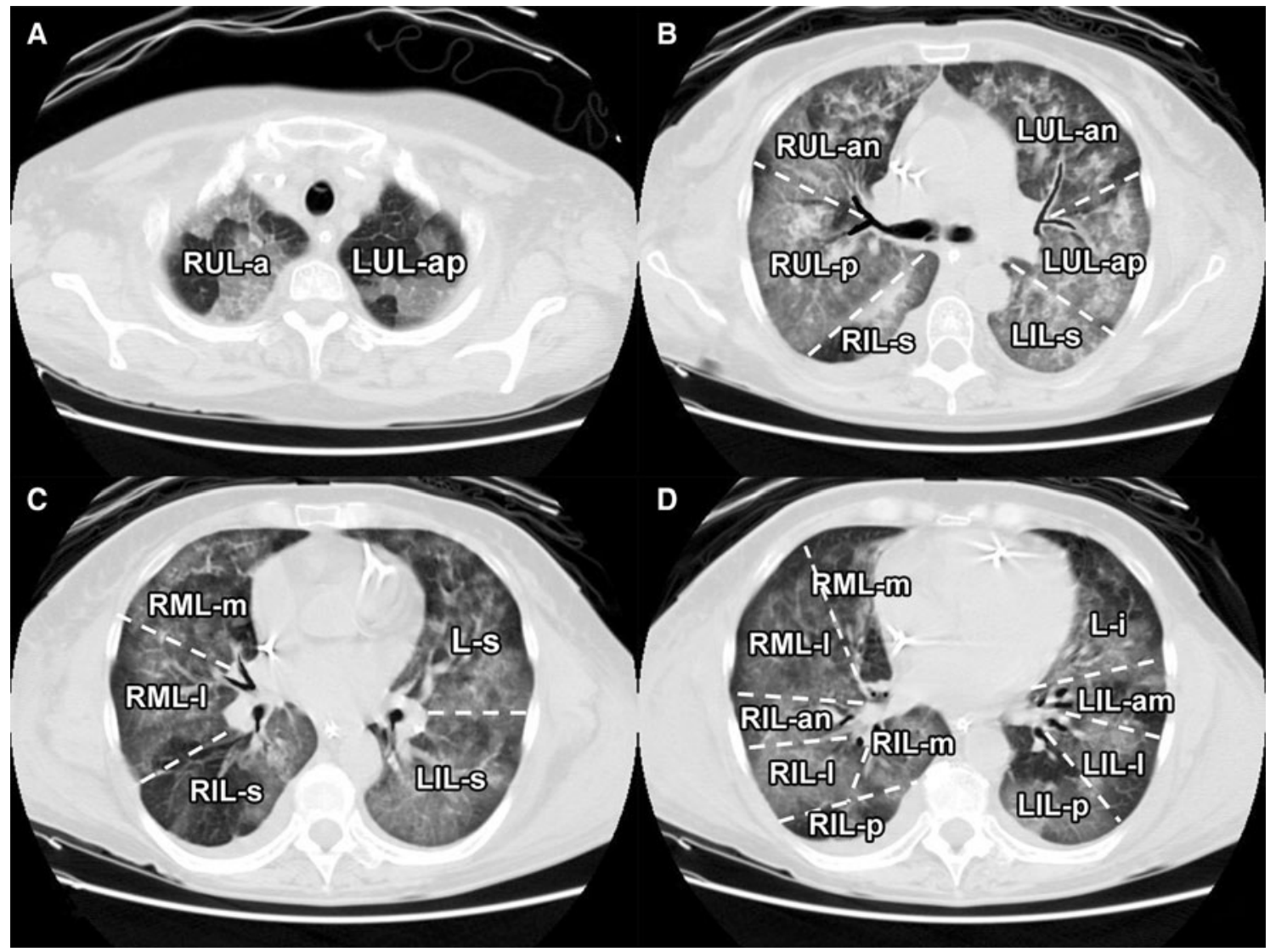

Fig. 1 Visual identification of the bronchopulmonary segments in the lung CT images. a $R U L-a$ right upper lobe, apical segment; $L U L$-ap left upper lobe, apical-posterior segment. b $R U L$-an right upper lobe, anterior segment; $R U L-p$ right upper lobe, posterior segment; RIL-s right inferior lobe, superior segment. $L U L$-an left upper lobe, anterior segment; $L U L$-ap left upper lobe, apicalposterior segment; $L I L-s$ left inferior lobe, superior segment. c $R M L-m$ right medium lobe, medial segment; $R M L-l$ right medium lobe, lateral segment; $R I L-s$ right inferior lobe, superior segment.
$L-s$ lingula, superior segment; $L I L-s$ left inferior lobe, superior segment. d $R M L-m$ right medium lobe, medial segment; $R M L-l$ right medium lobe, lateral segment; RIL-an right inferior lobe, anterior segment; $R I L-m$ right inferior lobe, medial segment. $R I L-p$ right inferior lobe, posterior segment; RIL-l right inferior lobe, lateral segment. $L-i$ lingula, inferior segment; LIL-am left inferior lobe, anteromedial segment; $L I L-l$ left inferior lobe, lateral segment; LIL- $p$ left inferior lobe, posterior segment 
cranial $50 \%$ is the superior segment whereas the remaining is the inferior segment (Fig. 1c, d).

\section{Quantitative lung CT scan analysis}

By using a custom designed software package, each crosssectional lung CT image was manually delineated, excluding pleural effusions, hilar and mediastinal structures by two investigators [11]. The tissue weight of lung regions with different degrees of inflation was calculated [11]. The lung regions were classified as not aerated (density between +100 and -100 Hounsfield units (HU)), poorly aerated (density between -101 and $-500 \mathrm{HU}$ ), normally aerated (density between -501 and $-900 \mathrm{HU}$ ), and hyperinflated (density between -901 and $-1,000 \mathrm{HU}$ ).

The lung recruitability was computed as the ratio between the decrease in tissue weight of not aerated lung regions passing from an airway pressure of 5 to $45 \mathrm{cmH}_{2} \mathrm{O}$ and the total lung weight at $5 \mathrm{cmH}_{2} \mathrm{O}$ [11].

\section{Statistical analysis}

Data with normal distribution are expressed as mean \pm SD or as median in the case of not normal distribution. The interobserver agreement between the two radiologists was assessed using the kappa coefficient test [18]. The lung recruitability computed by the visual anatomical and by the quantitative analysis was compared according to Bland-Altman analysis together with the Passing-Bablok nonparametric regression and concordance correlation coefficient [19]. Receiver operating characteristics (ROC) curve was plotted and area under the curve (AUC) was estimated, which may vary from 0.5 (poor discrimination) to 1 (perfect discrimination) [20].

The accuracy between the two analyses was computed as follows: sensitivity $=$ [true positive/(true positive + false negative) $] ;$ specificity $=[$ true negative/(true negative + false positive) $]$; and diagnostic accuracy $=$ [(true positive + true negative)/(true positive + false negative + true negative + false positive)].

All statistical tests were performed with SAS(R) version 9.2 (SAS Institute Inc., Cary, NC, USA). With 50 patients it has been possible to demonstrate a proportional error between the two measurement methods of about 0.40 at a significance of 0.05 (two tailed) and a power of about 0.80 by means of a correlation analysis between the difference and the means of the two measures.

\section{Results}

Fifty patients were studied. Table 1 shows the main characteristics of the whole population and divided according to the distribution of consolidated/collapsed lung tissue in the lung (patchy and lobar distribution).

The interobserver agreement between the two radiologists for estimating lung recruitability lower and higher than $9 \%$ was moderate as attested by a kappa coefficient of 0.62 .

The lung recruitability computed by the visual anatomical and by the quantitative lung CT scan analysis was median $11.3 \%$ (interquartile range $7.39-16.41 \%$ ) and median $15.5 \%$ (interquartile range $8.18-21.43 \%$ ), respectively.

The time required for the visual anatomical and for the quantitative lung CT analysis ranged between $20-30 \mathrm{~min}$ and 5-6 h.

Correlation between the visual anatomical and quantitative lung CT scan analysis

The regression equation calculated according to PassingBablok method had an intercept of 0.01458 (95\% CI -1.4464 to 1.3162$)$ and a slope of $0.8308(95 \% \mathrm{CI}$ $0.7387-0.9404)$ for the visual anatomical and quantitative analysis (Fig. 2).

In the Bland-Altman analysis, the bias and agreement bands (between brackets) for the visual anatomical and the quantitative analysis were $-2.9 \%(-11.8$ to $+5.9 \%)$ (Fig. 3).

The concordance correlation coefficient (CCC) was 0.86162 (95\% CI 0.77911-0.91479), resulting in a sample variability of $37 \%$.

Considering the distribution of consolidation/collapse lung tissue, the bias and agreement bands for the visual anatomical and the quantitative analysis were $-2.4 \%$ $(-11.5$ to +6.6$)$ and $-3.6 \%(-11.8$ to 4.6$)$ for patients with lobar and patchy distribution, respectively.

The ROC curve showed that the optimal cutoff value for the visual anatomical analysis for predicting patients with high lung recruitability was $8.9 \%$ (area under the $\mathrm{ROC}$ curve $=0.9248,95 \% \mathrm{CI}=0.8550-0.9946$ ) (Fig. 4). Considering this cutoff, there were 24 true positives, six false positives, 19 true negatives, and one false negative; thus the sensitivity, specificity, and diagnostic accuracy were 0.96 (95\% CI 0.863-0.999), 0.76 (95\% CI $0.593-0.906)$, and 0.86 (95\% CI $0.756-0.941)$ respectively. The positive and negative likelihood ratios were 4.0 (95\% CI 1.982-8.072) and 0.053 (95\% CI $0.007-0.363)$.

\section{Discussion}

This study showed an acceptable accuracy of visual anatomical analysis in detecting patients with high and low recruitable lung. The use of this simple analysis could 
Table 1 Baseline characteristics of the study population

\begin{tabular}{|c|c|c|c|c|}
\hline Characteristics & $\begin{array}{l}\text { Overall population } \\
(n=50)\end{array}$ & $\begin{array}{l}\mathrm{LD} \\
(n=29)\end{array}$ & $\begin{array}{l}\text { PD } \\
(n=21)\end{array}$ & $p$ value \\
\hline Age (years) & $57 \pm 19$ & $58 \pm 19$ & $55 \pm 19$ & 0.580 \\
\hline Female sex, $n(\%)$ & $20(40)$ & $11(38)$ & $9(43)$ & 0.953 \\
\hline Body mass index $\left(\mathrm{kg} / \mathrm{m}^{2}\right)$ & $25.7 \pm 5.2$ & $26.8 \pm 5.6$ & $24.2 \pm 4.1$ & 0.074 \\
\hline Tidal volume (mL/kg of predicted body weight) & $7.4 \pm 1.4$ & $7.2 \pm 1.5$ & $7.8 \pm 1.2$ & 0.122 \\
\hline Minute ventilation (L/min) & $9.2 \pm 2.4$ & $9.2 \pm 2.0$ & $9.1 \pm 2.9$ & 0.888 \\
\hline Respiratory rate (breath/min) & $16.6 \pm 5.0$ & $16.3 \pm 4.6$ & $17.0 \pm 5.6$ & 0.730 \\
\hline PEEP $\left(\mathrm{cmH}_{2} \mathrm{O}\right)$ & $10.7 \pm 2.6$ & $10.7 \pm 2.8$ & $10.7 \pm 2.5$ & 0.888 \\
\hline Elastance of respiratory system $\left(\mathrm{cmH}_{2} \mathrm{O} / \mathrm{L}\right)$ & $24.8 \pm 8.1$ & $24.0 \pm 8.7$ & $25.9 \pm 7.1$ & 0.306 \\
\hline $\mathrm{PaO}_{2} / \mathrm{FiO}_{2}$ & $176 \pm 64$ & $172 \pm 62$ & $180 \pm 77$ & 0.660 \\
\hline $\mathrm{FiO}_{2}$ & $0.53 \pm 0.15$ & $0.54 \pm 0.15$ & $0.52 \pm 0.15$ & 0.659 \\
\hline $\mathrm{PaCO}_{2}(\mathrm{mmHg})$ & $43.3 \pm 9.1$ & $42.3 \pm 7.0$ & $44.6 \pm 11.5$ & 0.387 \\
\hline \multicolumn{5}{|l|}{ Type of lung injury, $n(\%)$} \\
\hline ALI & $16(32)$ & & & \\
\hline ARDS & $34(68)$ & & & \\
\hline \multicolumn{5}{|l|}{ Causes of lung injury, $n(\%)$} \\
\hline Pneumonia & $27(54)$ & $11(38)$ & $16(76)$ & 0.017 \\
\hline Aspiration & $2(4)$ & $2(7)$ & $0(0)$ & 0.503 \\
\hline Nonpulmonary sepsis & $15(30)$ & $12(41)$ & $3(14)$ & 0.080 \\
\hline Trauma & $6(12)$ & $4(14)$ & $2(10)$ & 1.00 \\
\hline Postoperative patients, $n(\%)$ & $7(14)$ & & & \\
\hline Intensive care mortality, $n(\%)$ & $18(36)$ & $10(34)$ & $8(38)$ & 0.922 \\
\hline
\end{tabular}

$L D$ lobar distribution, $P D$ patchy distribution, $B M I$ body mass index, $P E E P$ positive end-expiratory pressure, $\mathrm{PaO}_{2}$ partial pressure of oxygen, $\mathrm{FiO}_{2}$ inspired oxygen fraction, $\mathrm{PaCO}_{2}$ partial pressure of carbon dioxide, $A L I$ acute lung injury, ARDS acute respiratory distress syndrome

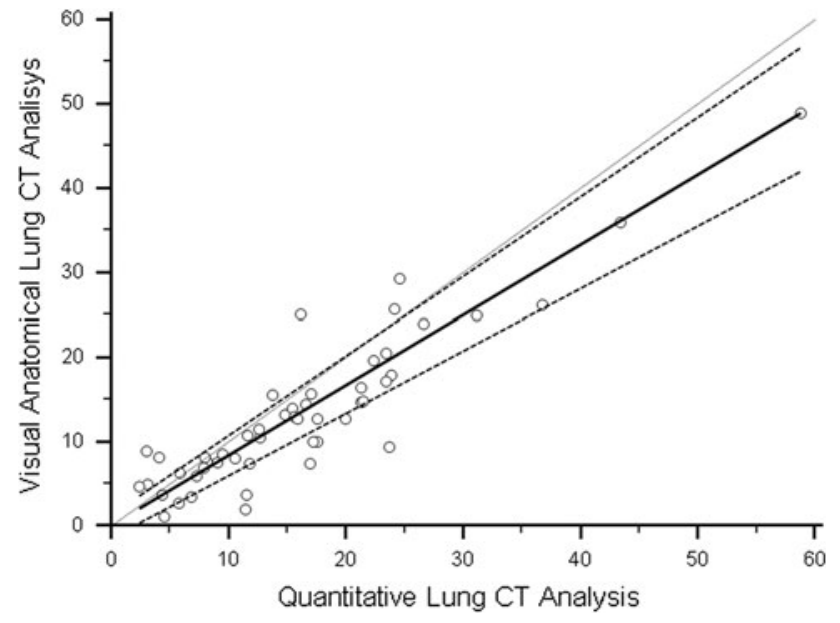

Fig. 2 Passing-Bablok regression considering lung recruitability with visual anatomical and quantitative lung CT analysis. Intercept of $0.01458(95 \% \mathrm{CI}-1.4464$ to 1.3162$)$ and slope of $0.8308(95 \%$ CI 0.7387-0.9404). Continuous line regression line, dashed lines confidence bands, dotted line identity line

allow a significantly wider use of lung CT to predict lung recruitability in patients with acute lung injury. The inflammatory edema in ARDS promotes alveolar collapse in the more dependent lung regions at various extensions [21]. By counterbalancing the increased compressive lung force, PEEP can limit opening/closing and alveolar collapse during the respiratory cycle, which have been recognized as factors promoting lung injury [22, 23]. Experimental animal studies, characterized by extensive

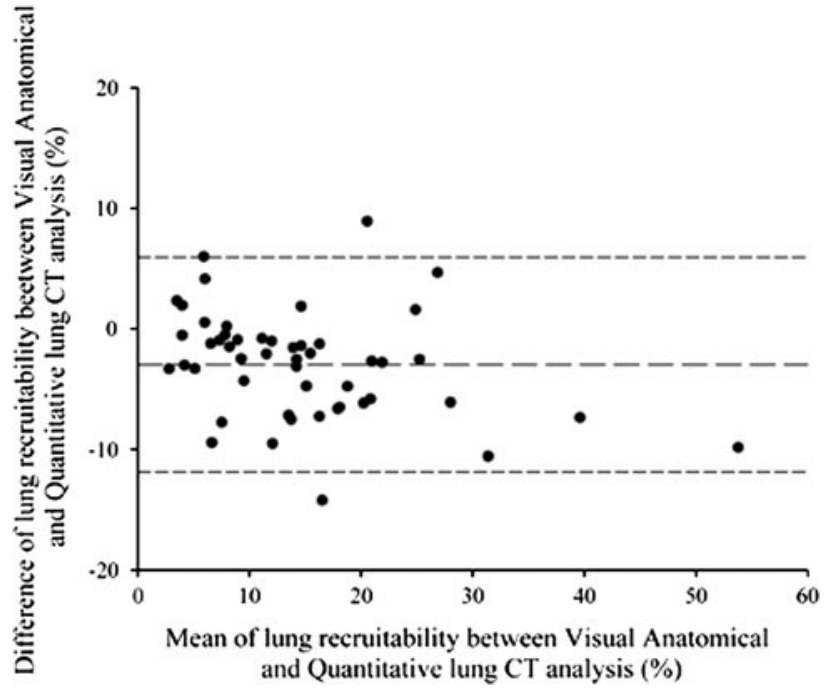

Fig. 3 Bland-Altman analysis of lung recruitability computed by the visual anatomical lung $\mathrm{CT}$ analysis and with the quantitative lung $\mathrm{CT}$ analysis. The $\mathrm{X}$-axis shows the mean of the two measurements and the Y-axis the difference between lung recruitability computed with visual anatomical lung CT analysis and with quantitative lung CT analysis

edema and collapse, showed that higher PEEP levels (i.e., $10-15 \mathrm{cmH}_{2} \mathrm{O}$ ) prevented ventilator-induced lung injury $[24,25]$. Conversely several randomized clinical trials failed to find any efficacy of higher PEEP compared to lower PEEP levels in patients with acute lung injury [26-28], suggesting that only a minority of these patients 


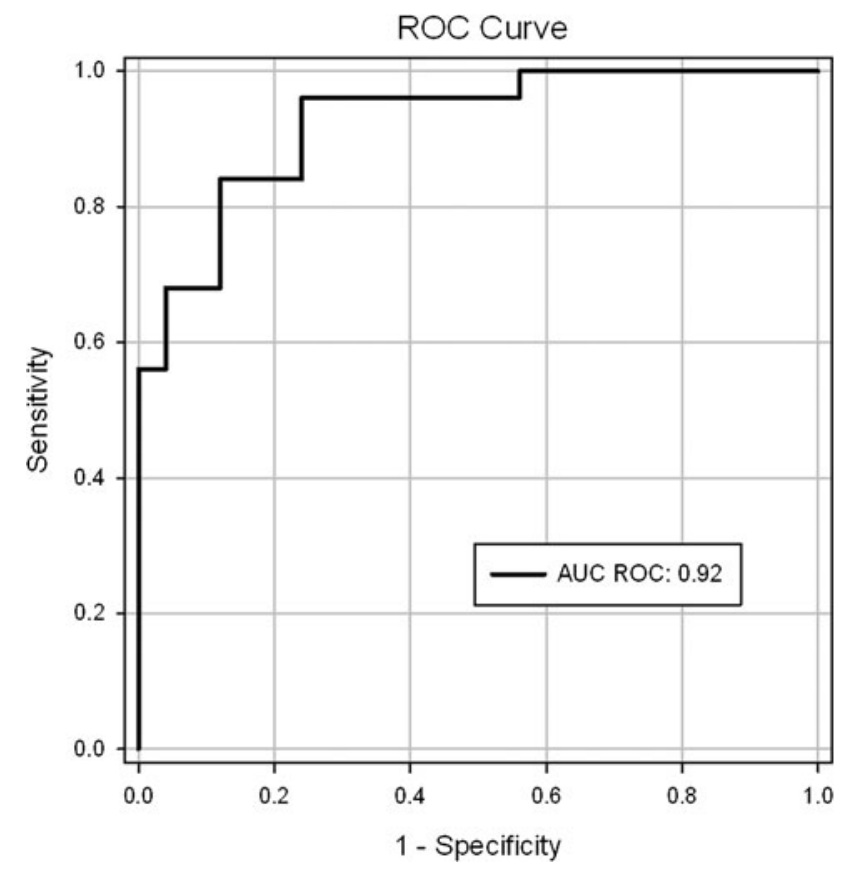

Fig. 4 Receiver operating characteristic curve for lung recruitability, mean visual anatomical lung CT scan analysis

are characterized by a great amount of alveolar collapse which could benefit from high PEEP levels [29]. In addition a recent study showed that high PEEP levels significantly decreased the opening and closing tissue without significantly increasing alveolar inflation only in patients with higher compared to lower lung recruitability [30]. Therefore the knowledge of lung recruitability may impact the mechanical ventilation setting [11].

Different methods such as the pressure-volume curve of the respiratory system [5,6], the use of electrical impedance tomography $[8,31]$, nitrogen washout/washin technique [32], and transthoracic lung ultrasound [9] have been proposed to estimate lung recruitability [4]. Although lung CT scan requires the transportation of patients and exposure to radiation, it remains the reference method for assessing lung recruitability [11]. Lung recruitability is computed using dedicated software [10, $11,33]$. However, contrary to healthy or emphysematous lung, in which an automatic segmentation of lung CT images is possible [34-36], in an ARDS lung a manual delineation separating the lung parenchyma from the pleural effusion, rib cage, or soft tissue is required because the software is not able to differentiate the not inflated tissue and pleural effusion because they present similar HU values. Considering a whole lung CT scan the number of images that must be delineated usually ranges from 40 to 60 , depending on the height of the subject, the thickness and the interval of the images. Thus the quantitative analysis is very laborious and time consuming, which precludes its use in the daily patient management
$[35,37,38]$. In order to expand the possibility to use the quantitative analysis to estimate lung recruitment, $\mathrm{Lu}$ et al. studied only one or three lung CT images instead of the whole lung CT scan, which required a significantly lower time [7]. However, the agreement was poor because of the inhomogeneous distribution of the disease in the lung parenchyma $[7,21]$.

Owing to the linear correlation between the CT attenuation and the density of the lung [37], we tested the accuracy of a visual assessment of percentage of consolidated/collapsed areas compared to a quantitative analysis. The consolidated/collapsed areas, which radiologically appear as areas of homogeneous decrease of lung attenuation, correspond to not aerated lung regions in the quantitative analysis. We used an upper limit of airway pressure of $45 \mathrm{cmH}_{2} \mathrm{O}$ because at this pressure almost $100 \%$ of opening pressure is reached [39]. On the contrary at lower airway pressures a lower estimation of lung recruitment would be obtained.

We found that agreement between the two operators performing the visual anatomical analysis was acceptable, although lower than those previously reported for the quantitative analysis $(<2 \%)$ [11]. However, a similar agreement was found by using the transthoracic lung ultrasound to detect lung recruitment [9] and to study the diaphragmatic motion [40]. This could be due to the high variability both in the anatomic localization of lobes and bronchopulmonary segments and in the visual assessment of the amount of consolidated/collapsed lung tissue in the CT images.

Although a relationship was found between the visual anatomical and the quantitative CT lung analysis in the present study, the Bland-Altman analysis showed that on average the visual anatomical analysis underestimated the recruitable lung compared to the lung CT scan and this error increased with increasing the recruitable lung. The limits of agreement ranged from -11.8 to $5.9 \%$. If we also divide patients according to a lobar or patchy distribution of the collapse/consolidation the accuracy of the visual anatomical analysis was similar. Similarly data were also found with the application of the transthoracic lung ultrasound in which the use of a visual reaeration score was able to predict with a sufficient accuracy the lung recruitability, dividing patients with high or low recruitability [9].

In clinical practice it is important to have a system of analysis which allows one to discriminate patients with high or low recruitable lung and not to compute the exact amount of recruitable lung which could translate into clinical practice by using lower PEEP in patients with low recruitability and conversely higher PEEP in patients with high recruitability. The specificity of the visual anatomical analysis was lower than the sensitivity causing a higher number of patients that would erroneously be identified as high recruiters. However, it would be better to set an inappropriately high PEEP in patients with low 
recruitability causing possible lung overdistension and hemodynamic impairment than the risk of not applying high PEEP in patients with high recruitability promoting intratidal collapse which is widely recognized as a damaging factor [11, 22, 23, 30, 41].

In a previous study using quantitative lung CT analysis, Gattinoni et al. [11] classified patients as higher or lower recruiters using the median value of recruitable lung which was $9 \%$. By using the present anatomical visual analysis, considering a cutoff value of $8.9 \%$ which is very similar to that used in the previous study [11], we were able to detect patients with higher and lower lung recruitability with good sensitivity and specificity. The sensitivity and specificity of the present study were significantly better than those obtained by computing the respiratory physiological variables (gas exchange, dead space, and respiratory compliance) to detect lung recruitment [11].

The limitations of visual anatomical analysis are: first, the interobserver variability of the operators in assessing the extension of the consolidated area in the bronchopulmonary segments. Second, the ability to detect the single segments of each lobe by recognizing the anatomic boundaries. Third, the difficulty of extrapolating the present results to all intensive care physicians because only two radiologists carried out the present analysis.
Fourth, the impossibility of estimating the change in the hyperinflated area, thus not allowing the computation of any form of overinflation. However, it must be stressed that even the quantitative lung CT scan analysis is not an ideal method to estimate the hyperinflation; in fact the traditional thresholds for the hyperinflated compartment may be hardly reached in the lung in which the baseline density is greatly increased as a result of the increased tissue mass.

\section{Conclusions}

Visual anatomical analysis can classify patients into those with a high or low recruitability with sufficient sensitivity and specificity. Hence this simple method could be used as an additional diagnostic tool to titrate a more physiologic mechanical ventilation. Further studies are needed to evaluate the benefit of selecting PEEP according to lung recruitability in terms of morbidity and outcome.

Acknowledgments This study was supported in part by an Italian grant provided by the Fondazione Fiera di Milano for Translational and Competitive Research.

\section{References}

1. The Acute Respiratory Distress Syndrome Network (2000) Ventilation with lower tidal volumes as compared with traditional tidal volumes for acute lung injury and the acute respiratory distress syndrome. N Engl J Med 342:1301-1308

2. Ranieri VM, Suter PM, Tortorella C, De Tullio R, Dayer JM, Brienza A, Bruno F, Slutsky AS (1999) Effect of mechanical ventilation on inflammatory mediators in patients with acute respiratory distress syndrome: a randomized controlled trial. JAMA 282:54-61

3. Lachmann B (1992) Open up the lung and keep the lung open. Intensive Care Med 18:319-321

4. Caironi P, Gattinoni L (2007) How to monitor lung recruitment in patients with acute lung injury. Curr Opin Crit Care 13:338-343

5. Ranieri VM, Giuliani R, Fiore T, Dambrosio M, Milic-Emili J (1994) Volume-pressure curve of the respiratory system predicts effects of PEEP in ARDS: "occlusion" versus "constant flow" technique. Am J Respir Crit Care Med 149:19-27
6. Thille AW, Richard JC, Maggiore SM, Ranieri VM, Brochard L (2007) Alveolar recruitment in pulmonary and extrapulmonary acute respiratory distress syndrome: comparison using pressure-volume curve or static compliance. Anesthesiology 106:212-217

7. Lu Q, Malbouisson LM, Mourgeon E, Goldstein I, Coriat P, Rouby JJ (2001) Assessment of PEEP-induced reopening of collapsed lung regions in acute lung injury: are one or three CT sections representative of the entire lung? Intensive Care Med 27:1504-1510

8. Costa EL, Borges JB, Melo A, SuarezSipmann F, Toufen C Jr, Bohm SH, Amato MB (2009) Bedside estimation of recruitable alveolar collapse and hyperdistension by electrical impedance tomography. Intensive Care Med 35:1132-1137

9. Bouhemad B, Brisson H, Le Guen M, Arbelot C, Lu Q, Rouby JJ (2011) Bedside ultrasound assessment of positive end-expiratory pressureinduced lung recruitment. Am J Respir Crit Care Med 183:341-347
10. Gattinoni L, Caironi P, Pelosi P, Goodman LR (2001) What has computed tomography taught us about the acute respiratory distress syndrome? Am J Respir Crit Care Med 164:1701-1711

11. Gattinoni L, Caironi P, Cressoni M, Chiumello D, Ranieri VM, Quintel M, Russo S, Patroniti N, Cornejo R, Bugedo G (2006) Lung recruitment in patients with the acute respiratory distress syndrome. N Engl J Med 354:1775-1786

12. Reske AW, Reske AP, Gast HA, Seiwerts M, Beda A, Gottschaldt U, Josten C, Schreiter D, Heller N, Wrigge H, Amato MB (2010) Extrapolation from ten sections can make CT-based quantification of lung aeration more practicable. Intensive Care Med 36:1836-1844

13. Bernard GR, Artigas A, Brigham KL, Carlet J, Falke K, Hudson L, Lamy M, Legall JR, Morris A, Spragg R (1994) The American-European consensus conference on ARDS. Definitions, mechanisms, relevant outcomes, and clinical trial coordination. Am J Respir Crit Care Med 149:818-824 
14. Puybasset L, Gusman P, Muller JC, Cluzel P, Coriat P, Rouby JJ (2000) Regional distribution of gas and tissue in acute respiratory distress syndrome. III. Consequences for the effects of positive end-expiratory pressure. CT Scan ARDS Study Group. Adult respiratory distress syndrome. Intensive Care Med 26:1215-1227

15. Austin JH, Muller NL, Friedman PJ, Hansell DM, Naidich DP, Remy-Jardin M, Webb WR, Zerhouni EA (1996) Glossary of terms for CT of the lungs: recommendations of the Nomenclature Committee of the Fleischner Society. Radiology 200:327-331

16. Pierce RJ, Brown DJ, Denison DM (1980) Radiographic, scintigraphic, and gas-dilution estimates of individual lung and lobar volumes in man. Thorax 35:773-780

17. Gutierrez FR, Rossi S, Bhalla S (2006) Thorax: techniques and normal anatomy. In: Lee JKT, Sagel SS, Stanley RJ, Heiken JP (eds) Computed body tomography with MRI correlation. Lippincott Williams and Wilkins, Philadelphia, pp 255-310

18. Sim J, Wright CC (2005) The kappa statistic in reliability studies: use, interpretation, and sample size requirements. Phys Ther 85:257-268

19. Chiumello D, Gallazzi E, Marino A, Berto V, Mietto C, Cesana B, Gattinoni L (2011) A validation study of a new nasogastric polyfunctional catheter. Intensive Care Med 37:791-795

20. Hanley JA, McNeil BJ (1982) The meaning and use of the area under a receiver operating characteristic (ROC) curve. Radiology 143:29-36

21. Pelosi P, D’Andrea L, Vitale G, Pesenti A, Gattinoni L (1994) Vertical gradient of regional lung inflation in adult respiratory distress syndrome. Am J Respir Crit Care Med 149:8-13

22. Mead J, Takishima T, Leith D (1970) Stress distribution in lungs: a model of pulmonary elasticity. J Appl Physiol 28:596-608

23. Muscedere JG, Mullen JB, Gan K, Slutsky AS (1994) Tidal ventilation at low airway pressures can augment lung injury. Am J Respir Crit Care Med 149:1327-1334

24. Dreyfuss D, Saumon G (1998) Ventilator-induced lung injury: lessons from experimental studies. Am J Respir Crit Care Med 157:294-323
25. Webb HH, Tierney DF (1974)

Experimental pulmonary edema due to intermittent positive pressure ventilation with high inflation pressures. Protection by positive end-expiratory pressure. Am Rev Respir Dis 110:556-565

26. Brower RG, Lanken PN, MacIntyre N, Matthay MA, Morris A, Ancukiewicz M, Schoenfeld D, Thompson BT (2004) Higher versus lower positive endexpiratory pressures in patients with the acute respiratory distress syndrome. N Engl J Med 351:327-336

27. Meade MO, Cook DJ, Guyatt GH, Slutsky AS, Arabi YM, Cooper DJ, Davies AR, Hand LE, Zhou Q, Thabane L, Austin P, Lapinsky S, Baxter A, Russell J, Skrobik Y, Ronco JJ, Stewart TE (2008) Ventilation strategy using low tidal volumes, recruitment maneuvers, and high positive endexpiratory pressure for acute lung injury and acute respiratory distress syndrome: a randomized controlled trial. JAMA 299:637-645

28. Mercat A, Richard JC, Vielle B, Jaber S, Osman D, Diehl JL, Lefrant JY, Prat G, Richecoeur J, Nieszkowska A, Gervais C, Baudot J, Bouadma L, Brochard L (2008) Positive endexpiratory pressure setting in adults with acute lung injury and acute respiratory distress syndrome: a randomized controlled trial. JAMA 299:646-655

29. Gattinoni L, Caironi P (2008) Refining ventilatory treatment for acute lung injury and acute respiratory distress syndrome. JAMA 299:691-693

30. Caironi P, Cressoni M, Chiumello D, Ranieri M, Quintel M, Russo SG, Cornejo R, Bugedo G, Carlesso E, Russo R, Caspani L, Gattinoni L (2010) Lung opening and closing during ventilation of acute respiratory distress syndrome. Am J Respir Crit Care Med 181:578-586

31. Meier T, Luepschen H, Karsten J, Leibecke T, Grossherr M, Gehring H, Leonhardt S (2008) Assessment of regional lung recruitment and derecruitment during a PEEP trial based on electrical impedance tomography. Intensive Care Med 34:543-550

32. Dellamonica J, Lerolle N, Sargentini C, Beduneau G, Di Marco F, Mercat A, Richard JC, Diehl JL, Mancebo J, Rouby JJ, Lu Q, Bernardin G, Brochard L (2011) PEEP-induced changes in lung volume in acute respiratory distress syndrome. Two methods to estimate alveolar recruitment. Intensive Care Med 37:1595-1604
33. Malbouisson LM, Preteux F, Puybasset L, Grenier P, Coriat P, Rouby JJ (2001) Validation of a software designed for computed tomographic (CT) measurement of lung water. Intensive Care Med 27:602-608

34. Coxson HO, Mayo J, Lam S, Santyr G, Parraga G, Sin DD (2009) New and current clinical imaging techniques to study chronic obstructive pulmonary disease. Am J Respir Crit Care Med 180:588-597

35. Madani A, De MV, Zanen J, Gevenois PA (2007) Pulmonary emphysema: radiation dose and section thickness at multidetector CT quantificationcomparison with macroscopic and microscopic morphometry. Radiology 243:250-257

36. Zaporozhan J, Ley S, Weinheimer O, Eberhardt R, Tsakiris I, Noshi Y, Herth F, Kauczor HU (2006) Multi-detector CT of the chest: influence of dose onto quantitative evaluation of severe emphysema: a simulation study. J Comput Assist Tomogr 30:460-468

37. Rouby JJ, Puybasset L, Nieszkowska A, Lu Q (2003) Acute respiratory distress syndrome: lessons from computed tomography of the whole lung. Crit Care Med 31:S285-S295

38. Simon BA (2000) Non-invasive imaging of regional lung function using X-ray computed tomography. J Clin Monit Comput 16:433-442

39. Crotti S, Mascheroni D, Caironi P, Pelosi P, Ronzoni G, Mondino M, Marini JJ, Gattinoni L (2001) Recruitment and derecruitment during acute respiratory failure: a clinical study. Am J Respir Crit Care Med 164:131-140

40. Boussuges A, Gole Y, Blanc P (2009) Diaphragmatic motion studied by m-mode ultrasonography: methods, reproducibility, and normal values. Chest 135:391-400

41. Oeckler RA, Hubmayr RD (2008) Cell wounding and repair in ventilator injured lungs. Respir Physiol Neurobiol 163:44-53 